\title{
Asimetría direccional en el cráneo del conejo de compañía
}

\section{Skull directional asymmetry in companion rabbit}

\author{
P.M. Parés-Casanova ${ }^{1,3}$, A. Medina ${ }^{2}$
}

\section{Resumen}

\begin{abstract}
La asimetría fluctuante se caracteriza por desviaciones sutiles y aleatorias en rasgos bilateralmente simétricos, y la asimetría direccional por una distribución sesgada hacia uno de los lados (derecho o izquierdo). Para el análisis de ellas en el cráneo del conejo de compañía, se estudiaron 45 especímenes. La morfología craneal fue revelada mediante el registro de coordenadas cartesianas de 24 hitos anatómicos sagitales (8) y bilaterales (en ambos lados del cráneo, 16), a nivel ventral, y se aplicaron métodos de morfometría geométrica. Los resultados indican diferencias entre las configuraciones derecha e izquierda del cráneo, sobre todo a nivel de la base. Esto podría interpretarse como una manifestación de la actividad masticatoria lateralizada.
\end{abstract}

Palabras clave: asimetría fluctuante; inestabilidad del desarrollo; pedomorfia; tamaño craneal

\section{Abstract}

Fluctuating asymmetry is characterized by subtle and random deviations in bilaterally symmetrical features, and directional asymmetry is characterized by a distribution skewed towards one side (left or right). For the analysis of both types in the skull of the companion rabbit, 45 specimens were studied. Cranial morphology was revealed by 8 sagittal and 16 bilateral (on both sides of the skull) Cartesian coordinates on the ventral level, using geometric morphometric. According to the results, there were differences between left and right configurations, located mainly on the basis. This fact may be interpreted as a manifestation of lateralized masticatory activity.

Key words: fluctuating asymmetry; developmental instability; pedomorphy; cranial size

${ }^{1}$ Departament de Ciència Animal, Universitat de Lleida, Lleida (Cataluña), España

2 Departamento de Ciencia y Producción Agropecuaria, Escuela Agrícola Panamericana El Zamorano, Honduras

${ }^{3}$ E-mail: peremiquelp@ca.udl.cat

Recibido: 9 de noviembre de 2018

Aceptado para publicación: 30 de mayo de 2019 


\section{INTRODUCCIÓN}

Una de las utilidades del análisis con métodos de morfometría geométrica es su capacidad de detectar alteraciones de la simetría (Mardia et al., 2000) (Briones y Guiñez, 2008). Entre los diversos tipos de asimetría se encuentra la asimetría fluctuante $(\mathrm{AF})$, caracterizada por desviaciones sutiles y aleatorias de la simetría en rasgos bilateralmente simétricos (Briones y Guiñez, 2008) y la asimetría direccional (AD), que se caracteriza por una distribución sesgada hacia uno de los lados (derecho o izquierdo) a nivel poblacional y que se origina como respuesta a estímulos externos que afectan diferencialmente a ambos lados del organismo (Lotto y Béguelin, 2014). La AD presenta una base genética significativa (Palmer, 1994) y, por lo tanto, sería apropiada para la estimación de procesos adaptativos.

El presente trabajo constituye pues una aproximación al análisis del grado y patrón de las dos asimetrías (AF y AD) en el cráneo del conejo de compañía, y particularmente dirigida a los diferentes módulos (esqueleto facial - esplacnocráneo - y encefálico neurocráneo-), en los que usualmente es dividido el cráneo en términos funcionales y del desarrollo.

\section{Materiales y Métodos}

Se estudiaron 45 especímenes de cráneos de conejo de compañía (tipo toy y belier) procedentes del mismo criador. Se utilizaron animales que fueron sacrificados en la misma granja por motivos ajenos a este trabajo. La morfología craneal fue relevada mediante el registro de coordenadas cartesianas de 24 hitos anatómicos sagitales (8) y bilaterales (en ambos lados del cráneo, 16) en 2 dimensiones (Figura 1). Las coordenadas fueron digitalizadas en los programas TPS-Util y TPS-Dig2 (Rohlf, 2015) y se obtuvo un archivo de extensión .tps con las coordenadas crudas. Los cráneos procedían de la colección del Departament de Ciència Animal de la Universitat de Lleida.

Puesto que la cuantificación de las asimetrías es altamente sensible al error de medición (EM) debido a que las desviaciones de la simetría son en general pequeñas y típicamente de similar magnitud que el EM (Lotto y Béguelin, 2014), se realizó un análisis multivariado de la varianza a partir de las coordenadas bilaterales del cráneo ventral del conjunto de coordenadas para cada individuo (tres réplicas a ciegas). El método de Superposición Procrustes Generalizado fue empleado para alinear las configuraciones de hitos $\mathrm{y}$, de este modo, eliminar la información sobre el tamaño, la posición y orientación original de las coordenadas cartesianas (Galan, 2016). La forma (i.e. shape), en este contexto, es definida como la información que resta en un conjunto de coordenadas después que estos parámetros han sido removidos (Klingenberg et al., 2002; Reyment, 2010). A partir de la superposición se extrajeron dos matrices; una que contenía el componente simétrico de la variación y la otra con el componente asimétrico. El primero representa la variación en forma entre los individuos y puede considerarse como el promedio de los lados derecho e izquierdo. El componente asimétrico fue estimado a partir de los hitos bilaterales y obtenido como la diferencia entre las coordenadas a ambos lados del eje de simetría.

El modelo permite evaluar simultáneamente el efecto de la lateralidad (asimetría direccional; $\mathrm{AD}$ ) y la interacción individuo*lateralidad (asimetría fluctuante; AF), considerando el primer factor como un efecto fijo y el segundo como un efecto aleatorio. En el caso de asimetrías significativas, ambos lados fueron evaluados mediante un análisis multivariante de la varianza (MANOVA).

Para obtener las variables dependientes se efectuó primero un análisis de componentes principales (ACP) sobre las configuraciones de puntos de los lados derecho e izquierdo de cada individuo superpuestas de 


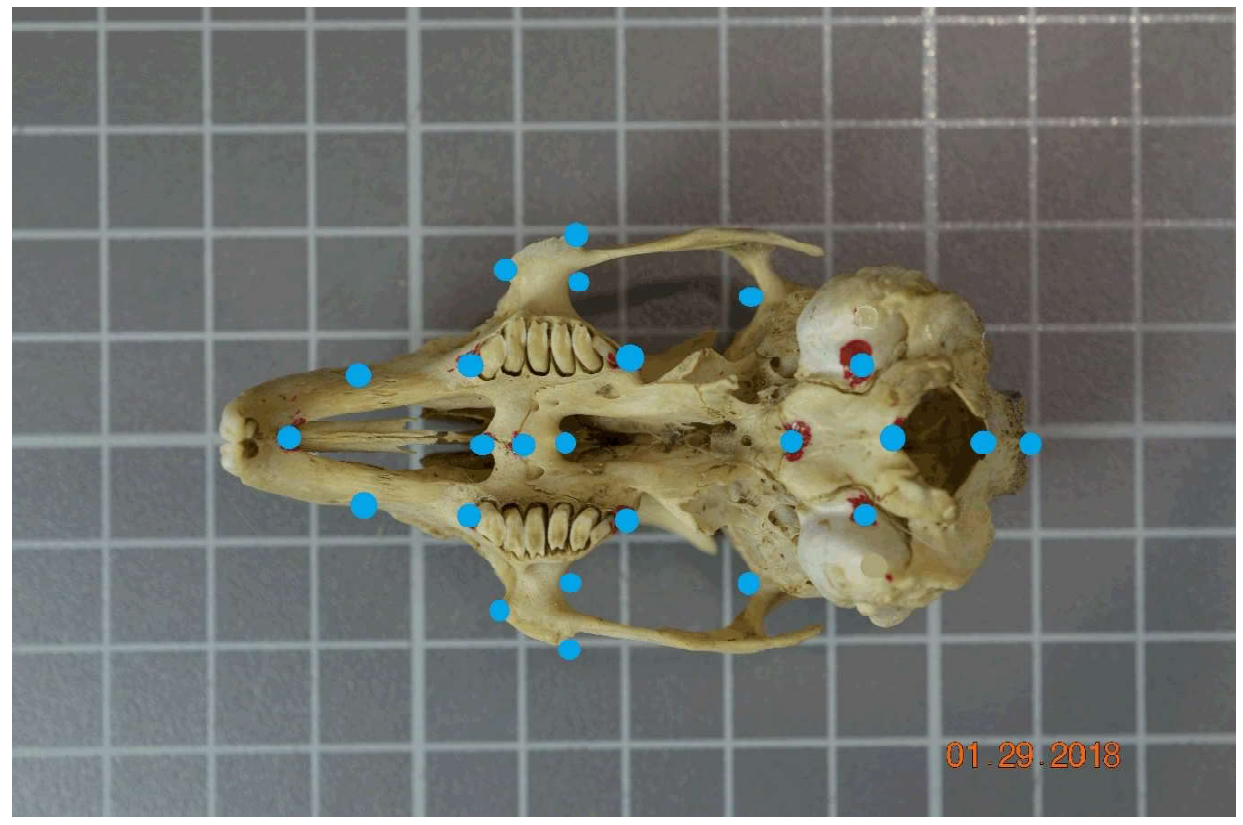

Figura 1. Hitos anatómicos utilizados. La morfología craneal fue relevada mediante el registro de coordenadas cartesianas de 24 hitos anatómicos sagitales (8) y bilaterales (en ambos lados del cráneo, 16) en 2 dimensiones

forma conjunta, y posteriormente sobre las configuraciones del esplacnocráneo y del neurocráneo por separado. Este procedimiento implica: 1) dividir cada configuración de puntos por su eje de simetría, repitiendo en ambas los puntos sagitales, 2) reflejar uno de los lados sobre su eje de simetría (e.g. invirtiendo el signo de las coordenadas), 3) superponer juntas las configuraciones de ambos lados de todos los individuos, y 4) estimar la matriz de covarianza sobre variación total (componente simétrico más asimétrico juntos) y el ACP. Se realizó finalmente una regresión de los componentes del esplacnocráneo y del viscerocráneo por separado, utilizando como variable independiente el tamaño de centroide logarítmicamente transformado.
La superposición, el ACP y las regresiones fueron realizados con el programa MorphoJ (Klingenberg, 2011), y el resto de los análisis con el programa PAST (Hammer et al., 2001). El valor de significación fue $\alpha=0.05$.

\section{Resultados}

Los resultados del ANOVA Procrustes se resumen en el Cuadro 1. La AD (lado), pero no la AF (interacción individuo*lado) resultó significativa $(\mathrm{p}<0.005)$, siendo la variación debida a AF mayor que la debida al error de medición. La variación explicada por AF alcanzó el 3.7\% del total y por AD el $33.9 \%$. Estos resultados justifican posterio- 
Cuadro 1 Resultados del ANOVA Procrustes

\begin{tabular}{llllll}
\hline Efecto & $\mathrm{SC}$ & $\mathrm{CM}$ & $F$ & $\mathrm{~g} .1$. & $P$ \\
\hline Individuo & 0.30344 & 0.000313 & 968 & 16.51 & $<0.0001$ \\
Lado & 0.00383 & 0.000174 & 22 & 9.19 & $<0.0001$ \\
Individuo * Lado & 0.01838 & $1.9 \mathrm{E}-05$ & 968 & 1.86 & 0.3034 \\
Error 1 & 0.02019 & $1.02 \mathrm{E}-05$ & 1980 & -2.63 & \\
Residual & -0.00768 & $-3.9 \mathrm{E}-06$ & 1980 & & \\
\hline
\end{tabular}

SC: suma de cuadrados; CM: cuadrados medios; F: valor del estadístico F; g.l.: grados de libertad El efecto "lado" resultó significativo $(p<0.005)$, siendo la variación debida a la interacción "individuo * lado" mayor que la debida al error de medición

res análisis para evaluar la magnitud de $\mathrm{AD}$ entre regiones del cráneo. De acuerdo a los resultados del MANOVA realizado sobre componentes principales de la variación entre lados, aparecieron diferencias entre muestras y entre las configuraciones derecha e izquierda del cráneo ventral $F(32.237)=$ 7066; $\mathrm{p}<0.01 ; \lambda$ de Wilk=0.0010).

La comparación de la dirección de los vectores de forma del componente asimétrico en el cráneo completo mostró que el primer componente principal (CP1) explicaba únicamente el $26.7 \%$ de la varianza total. La configuración espacial del CP1 mostró la asimetría principalmente en la región de la base. La regresión se mostró para los componentes de viscerocráneo $(\mathrm{p}=0.0016)$, pero no del neurocráneo $(\mathrm{p}=0.769)$, con una variación explicada para el primer componente claramente mayor $(2.54 \%$ vs. $0.86 \%)$.

\section{Discusión}

Varios autores sugieren que la combinación de múltiples rasgos de simetría bilateral en una muestra aumenta la posibilidad de detectar asimetría en una población (Nuffel et al., 2007). En este sentido, el empleo de técnicas de morfometría geométrica permite registrar y analizar conjuntos de rasgos de manera simultánea manteniendo la configuración espacial de las estructuras estudiadas. Los resultados obtenidos en los análisis preliminares indicaron que el error de medición es bajo con relación a la magnitud de la AF. En consecuencia, para un campo de estudio en el que el tamaño de la muestra está limitado por factores ajenos al diseño, se considera que la relación entre número de individuos y repeticiones utilizadas en el presente trabajo resulta adecuada para obtener estimaciones confiables de las asimetrías. La muestra presentó, además, niveles no significativos de $\mathrm{AF}$ en relación con la varianza total (3.7\%), pero no de $\mathrm{AD}(33.9 \%)$, que resultó significativa. Esto permitió evitar el problema derivado de un aumento artificial de los valores en los índices de $\mathrm{AD}$ debidos a la $\mathrm{AF}$, que por lo tanto, la AF no constituiría un factor relevante en la estimación del componente asimétrico de la variación en forma.

Asimismo, se observó un patrón disímil en las regiones del cráneo. Mientras que la región facial no presentó muchas diferencias por lados, la magnitud de asimetría en la base fue mucho más elevada. Esta elevada magnitud de asimetría en la base del cráneo sugiere que los procesos que dieron origen a la 
asimetría observada en esta estructura deben haber tenido una intensidad similar en todas las muestras.

Cabe destacar que una presunción común en la literatura es que el desarrollo de los lados izquierdo y derecho procede independientemente (Briones y Guiñez, 2008). Sin embargo, los resultados del estudio permiten indicar que, por lo menos en el caso considerado, se daría una retroalimentación entre los dos lados de un rasgo, lo cual llevaría a mecanismos de crecimiento compensatorio diferencial entre ambos lados. A diferencia de la $\mathrm{AF}$, en que los efectos de las perturbaciones en los procesos de desarrollo son acumulativos a lo largo del tiempo, en la AD no sería de esperar encontrar una asociación entre la duración del período de crecimiento y la magnitud de la asimetría. Por eso, aunque los elementos condrales de la base del cráneo sigan un patrón de crecimiento caracterizado por un temprano y rápido aumento del tamaño, y las estructuras faciales completen su desarrollo a una edad más avanzada, es en la primera donde la AD no cambia con el tamaño.

El hueso es un tejido dinámico, que experimenta un remoldeado adaptativo; es decir, una reabsorción y una aposición continuas, para satisfacer las necesidades de su entorno funcional. Sería una lateralización de esta tasa, dentro de un proceso de remodelación derivado de una diferente actividad masticatoria en ambos lados, la que lo explicaría (por los músculos pterigoide medial y masetero superficial) (Barone, 2000). En condiciones fisiológicas, efectivamente, los músculos proporcionan así un estímulo mecánico importante para la remodelación ósea mediante la inducción de tensiones en el sistema esquelético (Franchi et al., 2017). Por ello esta unilateralidad podría interpretarse como una manifestación de la actividad masticatoria lateralizada. Otros posibles factores, como el de un diferente desarrollo centrado en diferencias en el aporte vascular parecería muy poco probable. Además, exis- te bibliografía que detecta asimetría direccional por fenómenos de lateralización masticatoria en otras especies domésticas, como el caballo (Parés-Casanova, 2014b), ovino (Parés-Casanova y Bravi, 2014) y suidos (Parés-Casanova, 2014a), de allí que lo detectado en el conejo no es más que una corroboración de un fenómeno que parecería fisiológico en los animales.

Estudios adicionales de la masa del músculo del masticatorio en relación con la forma craneal permitirían aclarar las contribuciones del desarrollo muscular, contracción y cargamento biomecánico en lagomorfos.

\section{Conclusiones}

Los cráneos de los conejos de compañía presentan niveles significativos de asimetría direccional en el plano ventral, probablemente debido a una lateralización masticatoria. Corroboraría ello que el fenómeno de direccionalidad a nivel craneal es muy común entre las especies de mamíferos domésticos.

\section{Agradecimientos}

Los autores agradecen encarecidamente a la empresa CUNIPIC, de Térmens (Cataluña), por su interés en la entrega de los cadáveres, $\mathrm{y}$ ofrecer en todo momento la información que se les podía haber requerido.

\section{Literatura Citada}

1. Barone R. 2000. Anatomie comparée des mammifères domestiques. Vol 2. Artrhologie et myologie. Paris: Vigot.

2. Briones C, Guiñez R. 2008. Una revisión de la asimetría bilateral en bivalvos. Rev Biol Mar Oceanog 43: 1-6. doi: 10.4067/S0718-19572008000100001

3. Franchi MV, Reeves ND, Narici MV. 2017. Skeletal muscle remodeling in- 
response to eccentric vs. concentric loading: morphological, molecular, and metabolic adaptations. Front Physiol 8: 447. doi: 10.3389/fphys.2017.00447

4. Galan AL. 2016. Morfometría geométrica: el estudio de la forma y su aplicación en biología. Temas de Ciencia y Tecnología 19: 53-59.

5. Hammer Ø, Harper DAT, Ryan PD. 2001. PAST v. 2.17 c. Palaeontologia Electronica 4: 1-229.

6. Klingenberg CP. 2011. MorphoJ: An integrated software package for geometric morphometrics. Mol Ecol Resour 11: 353-357. doi: 10.1111/j.17550998.2010.02924.x

7. Klingenberg CP, Barluenga M, Meyer A. 2002. Shape analysis of symmetric structures: Quantifying variation among individuals and asymmetry. Evolution 56: 1909-1920. doi: 10.1111/j.00143820.2002.tb00117.x

8. Lotto F, Béguelin M. 2014. Asimetría direccional del postcráneo en poblaciones prehispánicas del sur de Sudamérica. Antropol Biol 7 133-142.

9. Mardia KV, Bookstein FL, Moreton IJ. 2000. Statistical assessment of bilateral symmetry of shapes. Biometrika 87: 285-300. doi: 10.1093/biomet/87.2.285
10. Nuffel AV, Tuyttens FAM, Dongen SV, Talloen W, Poucke EV, Sonck B, Lens L. 2007. Fluctuating asymmetry in broiler chickens: a decision protocol for trait selection in seven measuring methods. Poultry Sci 86: 2555-2568. doi: 10.3382/ps.2006-00192

11. Palmer AR. 1994. Fluctuating asymmetry analysis: a primer. In: Conference on developmental instability: its origins and evolutionary implications. Temple, Arizona, USA.

12. Parés-Casanova PM. 2014a. Existence of mandibular directional asymmetry in the European wild boar (Sus scrofa Linnaeus, 1758). J Morphol Sci 31: 1-5. doi: $10.4322 /$ jms.064613

13. Parés-Casanova PM. 2014b. Size asymmetries in equine upper molar series. ECORFAN J 5: 2055-2069.

14. Parés-Casanova PM, Bravi R. 2014. Directional and fluctuating asymmetries in domestic sheep skulls. J Zool Bioscience Res 2: 11-17.

15. Reyment $R A$. 2010. Morphometrics: an historical essay. In: Morphometric for nonmorphometricians. Springer-Verlag Berlin Heidelberg. p 9-24

16. Rohlf FJ. 2015. The tps series of software. Hystrix 26: 9-12. doi: 10.4404/ hystrix-26.1-11264 